\title{
RR Lyrae stars as probes of the Milky Way structure and formation
}

\author{
Pawel Pietrukowicz and OGLE collaboration \\ Warsaw University Observatory, Al. Ujazdowskie 4, 00-478 Warszawa, Poland \\ pietruk@astrouw.edu.pl
}

\begin{abstract}
RR Lyrae stars being distance indicators and tracers of old population serve as excellent probes of the structure, formation, and evolution of our Galaxy. Thousands of them are being discovered in ongoing wide-field surveys. The OGLE project conducts the Galaxy Variability Survey with the aim to detect and analyze variable stars, in particular of RRab type, toward the Galactic bulge and disk, covering a total area of $3000 \mathrm{deg}^{2}$. Observations in these directions also allow detecting background halo variables and unique studies of their properties and distribution at distances from the Galactic Center to even $40 \mathrm{kpc}$. In this contribution, we present the first results on the spatial distribution of the observed RRab stars, their metallicity distribution, the presence of multiple populations, and relations with the old bulge. We also show the most recent results from the analysis of RR Lyrae stars of the Sgr dwarf spheroidal galaxy, including its center, the globular cluster M54.
\end{abstract}

Keywords. Galaxy: structure, Galaxy: formation, stars: variables: RR Lyrae

RR Lyrae stars are core helium-burning giants with theoretically estimated masses in a range from about 0.55 to $0.80 M_{\odot}$ and ages $>10$ Gyr (Marconi et al. 2015). These pulsating stars have spectral types from A2 to F6 or effective temperatures between 6500 and $9000 \mathrm{~K}$ and $V$-band absolute magnitudes in a range from +0.3 to $+0.9 \mathrm{mag}$. RR Lyrae stars can be found everywhere in our Galaxy. Thousands of them have been discovered in wide-field surveys such as: ASAS, Catalina, MACHO, NSVS, OGLE, PTF, QUEST, SDSS, SEKBO, VVV. RR Lyrae stars are divided into fundamental-mode (type RRab), first-overtone (type RRc), and rarely found double-mode (type RRd) pulsators. RRab stars are on average intrinsically brighter and have higher amplitudes than RRc stars. More importantly, RRab variables with their characteristic saw-shaped light curves, in comparison to nearly-sinusoidal light curves of RRc stars, are hard to overlook making the searches for this type of variables highly complete. RRab stars have also a very practical photometric property. Based on the pulsation period and shape of the light curve one can estimate the metallicity of the star (Jurcsik 1995; Jurcsik \& Kovács 1996; Smolec 2005).

The OGLE project (the Optical Gravitational Lensing Experiment) is a long-term variability survey which started in 1992 with the original aim to detect microlensing events toward the Galactic bulge (Udalski et al. 1992). Since the installation of a 32chip camera with $1.4 \mathrm{deg}^{2}$ field of view in 2010 , the project has been in its fourth phase (OGLE-IV, Udalski et al. 2015) and focuses on large-scale monitoring. Currently, OGLE monitors about 1.3 billion stars located in dense regions of the sky such as the Galactic bulge, Galactic disk, and Magellanic Clouds, by covering a total area of over $3000 \mathrm{deg}^{2}$. The survey is conducted with the 1.3-m Warsaw telescope at Las Campanas Observatory, Chile, administrated by the Carnegie Institution for Science.

Recently, Soszyński et al. (2014) released a collection of 38,257 RR Lyrae stars detected in the OGLE-IV Galactic bulge fields. An analysis of the subset of 27,258 RRab stars has 


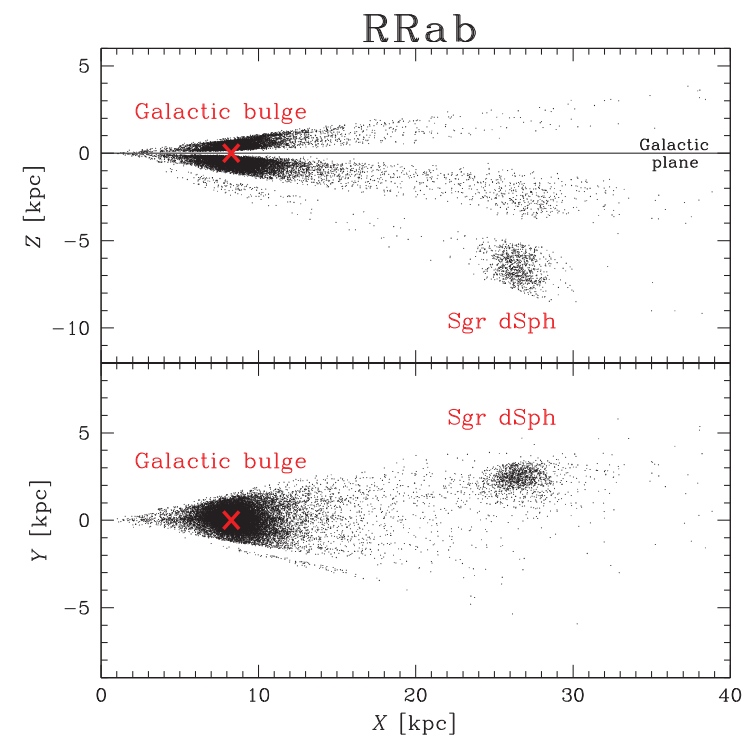

Figure 1. Projection of nearly 23,000 RRab stars observed toward the OGLE-IV bulge fields onto $X Z$ and $X Y$ planes. The Sun is located at the origin of the system. The variables are concentrated around the Galactic Center and distributed to the outer halo. The second concentration is formed of variables from the tidally disrupted Sgr dSph galaxy.

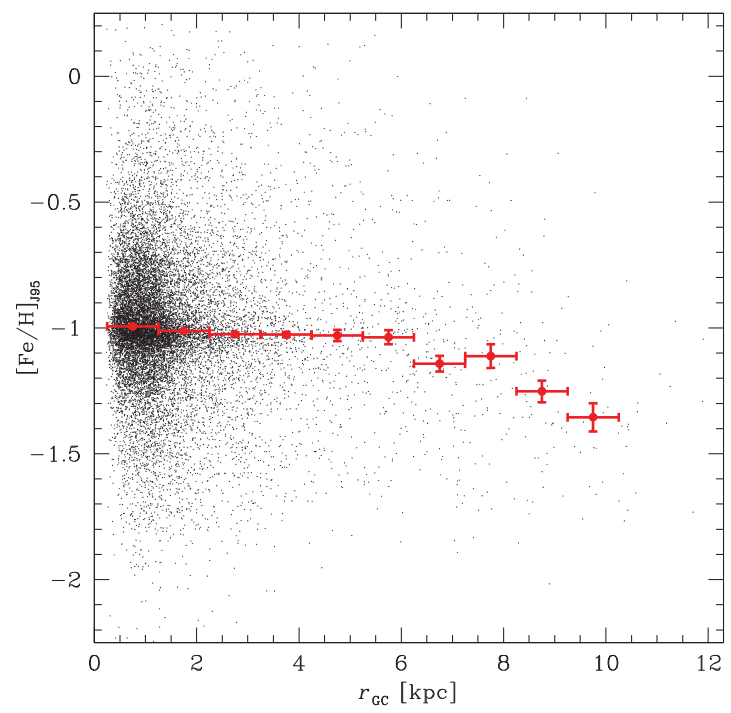

Figure 2. Metallicity distribution on the Jurcsik (1995) scale as a function of distance from the Galactic Center. Note a break around $6 \mathrm{kpc}$.

been published by Pietrukowicz et al. (2015). According to their work, these metal-poor stars trace closely the barred structure formed of intermediate-age red clump giants. The obtained distance to the Galactic center (GC) from the RR Lyrae stars is $R_{0}=$ $8.27 \pm 0.01$ (stat) \pm 0.40 (sys) kpc, in very good agreement with the most recent estimates of $R_{0}$ from other methods. They show that the spatial distribution has the shape of a triaxial ellipsoid with proportions 1:0.49(2):0.39(2) and the major axis located in the Galactic plane and inclined at an angle $i=20^{\circ} \pm 3^{\circ}$ to the Sun-GC line of sight. Another 


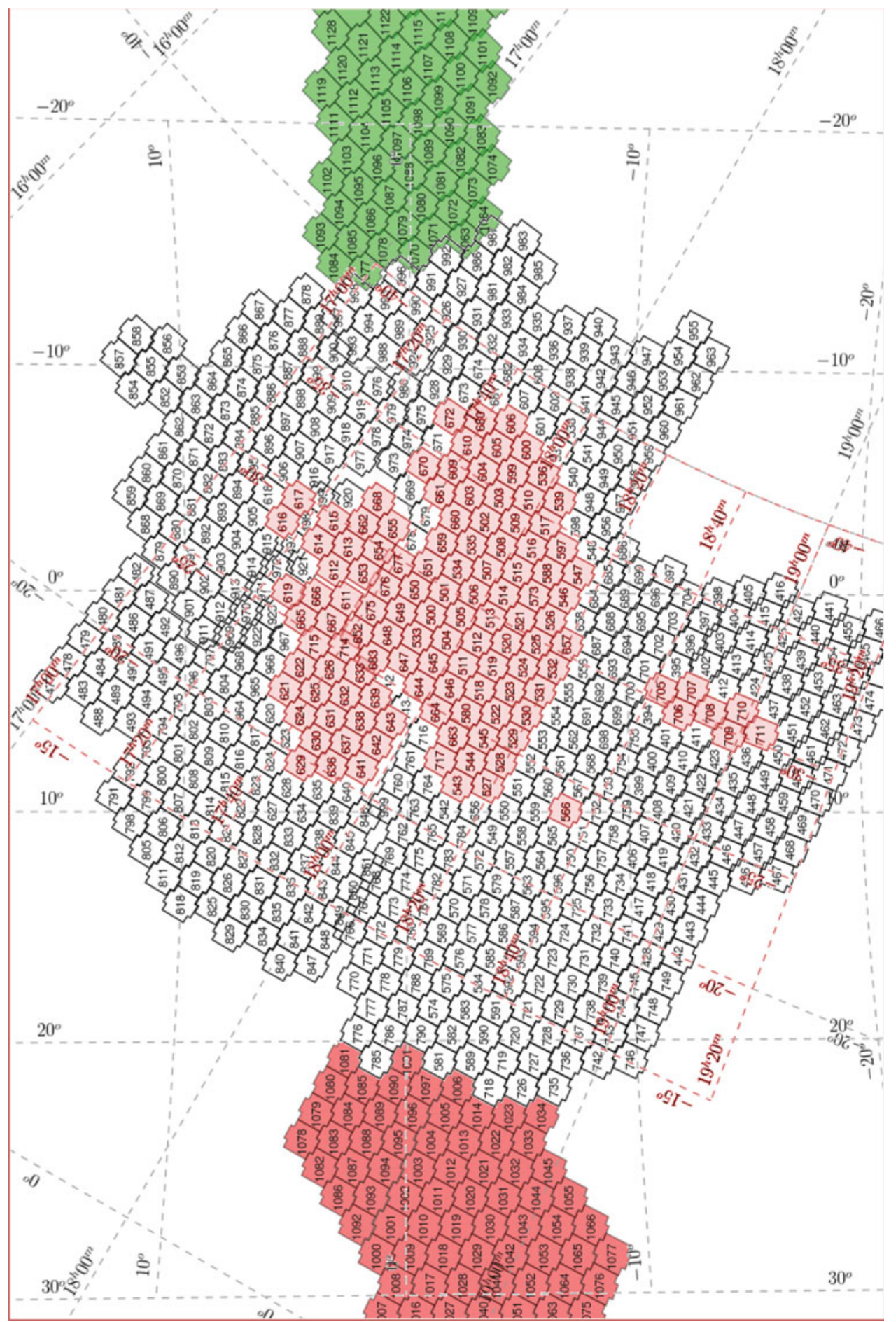

Figure 3. Extended coverage of the OGLE-IV bulge area. RR Lyrae stars from the central fields (in light pink) are analyzed in Pietrukowicz et al. (2015) and presented in this contribution. Fields at larger Galactic longitudes (in green and red) represent Galactic disk fields. 
discovery is the presence of multiple old populations being likely the result of mergers in the early history of the Milky Way.

In this contribution, we report important results on far RR Lyrae variables, namely stars observed behind the Galactic bulge. Fig. 1 shows that the distribution of stars from the Galactic bulge area to the outer halo is smooth. Pietrukowicz et al. (2015) has found that the spatial density profile of bulge RR Lyrae variables can be described as a single power law with an index of -2.96 . This value is very similar to indices obtained for halo stars, for example -2.8 for main-sequence stars near turn-off point (Jurić et al. 2008).

The prominent structure seen in Fig. 1 at a distance about three times larger than that to the GC is the remnant of the Sagittarius dwarf spheroidal (Sgr dSph) galaxy. Our search for variables within the tidal radius of M54, the globular cluster located at the core of Sgr dSph, has brought the detection of 277 such objects including 182 RR Lyrae stars, of which 23 are new. Based on 65 RRab stars, very likely members of the cluster, we have estimated the distance to M54 as $27.1 \pm 0.2$ (stat) \pm 1.3 (sys) kpc (Hamanowicz et al., in prep.).

In Fig. 2, we present the radial metallicity distribution for RRab stars from the GC out to about $12 \mathrm{kpc}$. The metallicity clearly decreases with the distance from the center but the decrease is very mild to about $6 \mathrm{kpc}$ and much more steeper farther out. At a distance of $8 \mathrm{kpc}$ from the GC it amounts to about -1.1 dex on the Jurcsik (1995) scale, in agreement with what is observed in the solar vicinity. For instance, a mean metallicity for 28 field RR Lyrae stars listed in Smolec (2005) is -1.05 dex.

Our results indicate that the old bulge and halo form one component of the Galaxy. Recently, in a sample of 100 RR Lyrae stars from the bulge area, Kunder et al. (2015) found a high velocity object on a halo-like orbit. Ongoing and future surveys will complete our knowledge on the shape and properties of the old component. The OGLE survey has extended the coverage of the bulge area with the prime aim to find and characterize RR Lyrae stars to an angular distance of about $22^{\circ}$ from the Galactic center (see Fig. 3).

\section{Acknowledgements}

The OGLE project has received funding from the National Science Centre, Poland, grant MAESTRO 2014/14/A/ST9/00121 to A. Udalski. This work has been also supported by the Polish Ministry of Sciences and Higher Education grants No. IP2012 005672 under the Iuventus Plus program to P. Pietrukowicz and No. IdP2012 000162 under the Ideas Plus program to I. Soszyński.

\section{References}

Marconi, M., Coppola, G., Bono, G., et al. 2015, ApJ, 808, 50

Jurić, M., Ivezić, Ž., Brooks, A., et al. 2008, ApJ, 673, 864

Jurcsik, J. 1995, AcA, 45, 653

Jurcsik, J. \& Kovács, G. 1996, A\&\&A, 312, 111

Kunder, A., et al. 2015, ApJL, 808, L12

Pietrukowicz, P., et al. 2015, ApJ, 811, 113

Smolec 2005, AcA, 55, 59

Soszyński, I., Udalski, A., \& Szymański, M. K., et al. 2014, AcA, 64, 177

Udalski, A., Szymański, M., Kaluzny, J., Kubiak, M., \& Mateo, M. 1992, AcA, 42, 253

Udalski, A., Szymański, M., \& Szymański, G. 2015, AcA, 65, 1 